Supporting Information for

\title{
Understanding the Loading Dependence of Adsorbate Diffusivities in Hierarchical Metal-Organic Frameworks
}

Haoyuan Chen and Randall Q. Snurr*

Department of Chemical \& Biological Engineering, Northwestern University, 2145 Sheridan

Road, Evanston, IL 60208, USA

\section{Convergence of the MD Simulations}

10 ns NVT equilibration, 55 n-hexane molecules in NU-1000

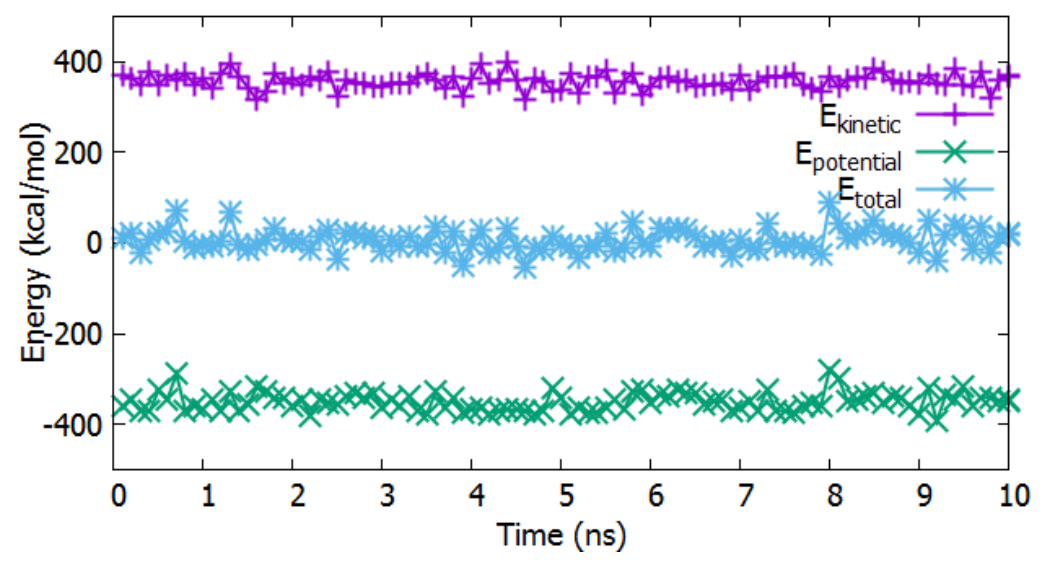

100 ns NVE production, 55 n-hexane molecules in NU-1000

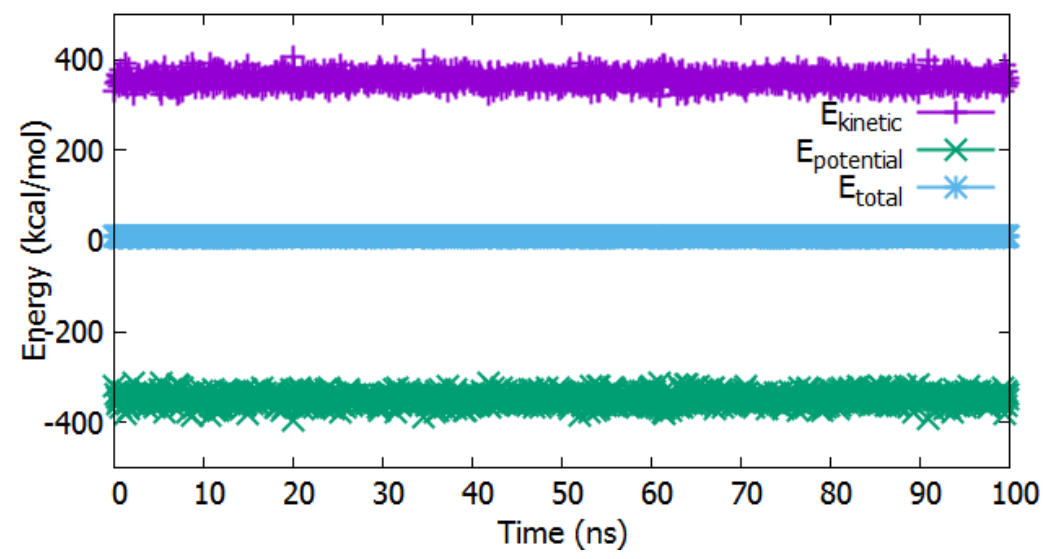

Figure S1. Convergence of kinetic, potential and total energies in one of the MD simulations (n-hexane in NU-1000 at $363 \mathrm{~K}$, loading $=55$ molecules per unit cell) during the equilibration (top) and production (bottom) stages. 


\section{Mean Square Displacement (MSD) - Time Lag Plots}
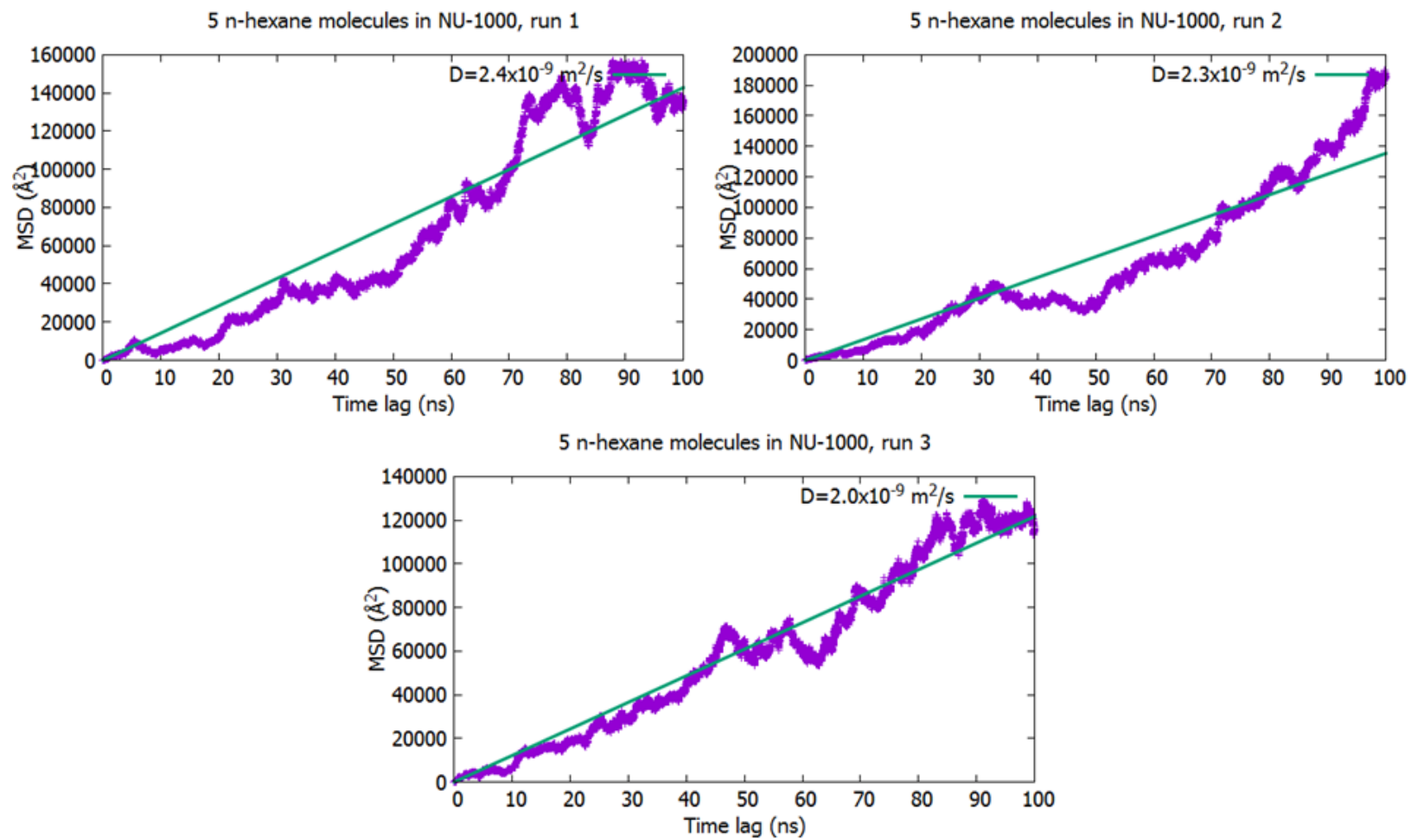

Figure S2. MSD plots of the 3 runs at one of the low loadings (loading $=5$ molecules per unit cell, nhexane in NU-1000 at $363 \mathrm{~K}$ ). The data have some deviations from linear due to statistical problems at low loading, but the self-diffusion coefficients obtained from the 3 runs are very consistent. All MSD values reported in this work were obtained using a single time origin (the starting point of the production run), as implemented in the LAMMPS package. 

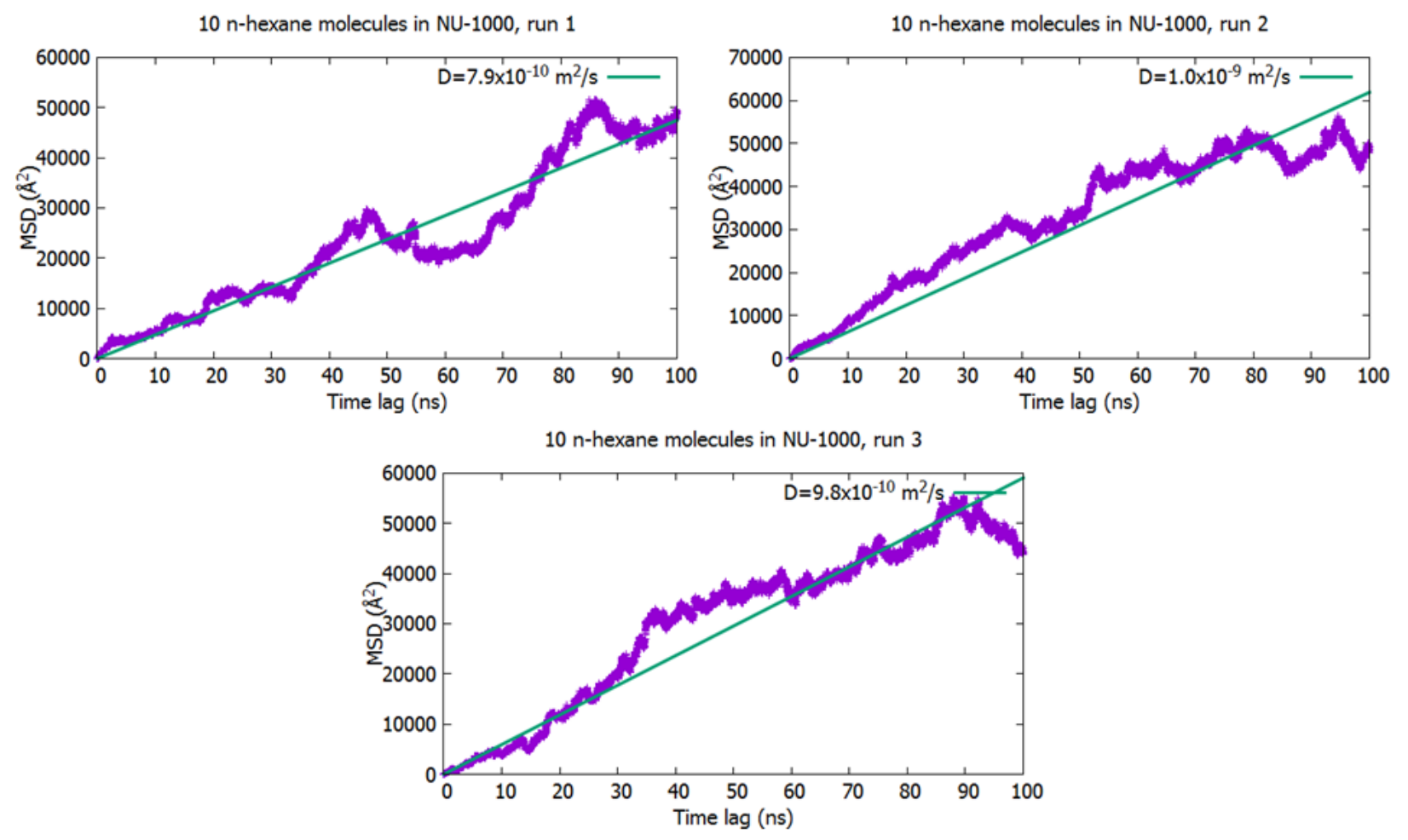

Figure S3. MSD plots of the 3 runs at one of the low loadings (loading $=10$ molecules per unit cell, nhexane in NU-1000 at $363 \mathrm{~K}$ ). The data have some deviations from linear due to statistical problems at low loading, but the self-diffusion coefficients obtained from the 3 runs are very consistent. 

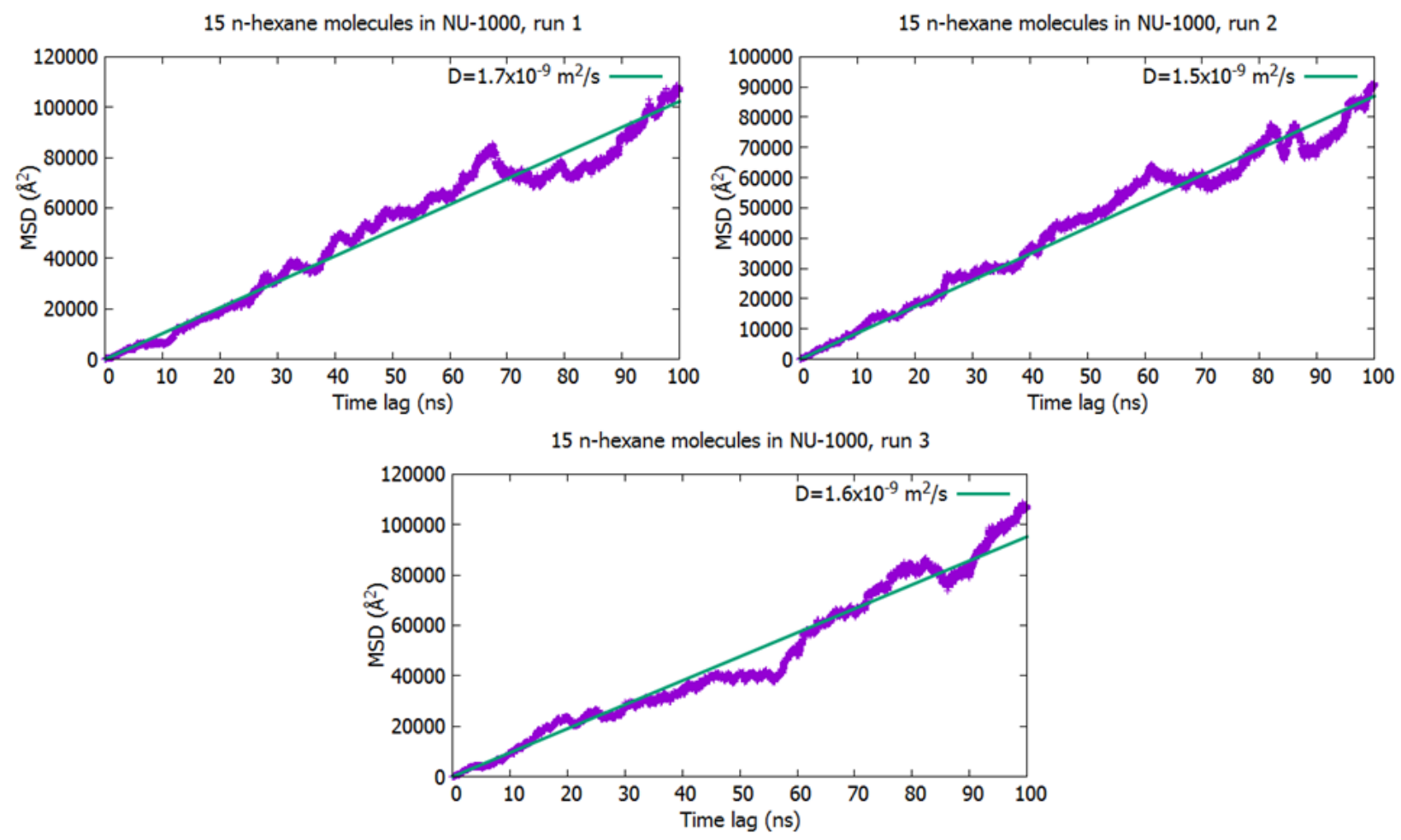

Figure S4. MSD plots of the 3 runs at one of the low loadings (loading $=15$ molecules per unit cell, $n$ hexane in NU-1000 at $363 \mathrm{~K}$ ). The data have some deviations from linear due to statistical problems at low loading, but the self-diffusion coefficients obtained from the 3 runs are very consistent. 

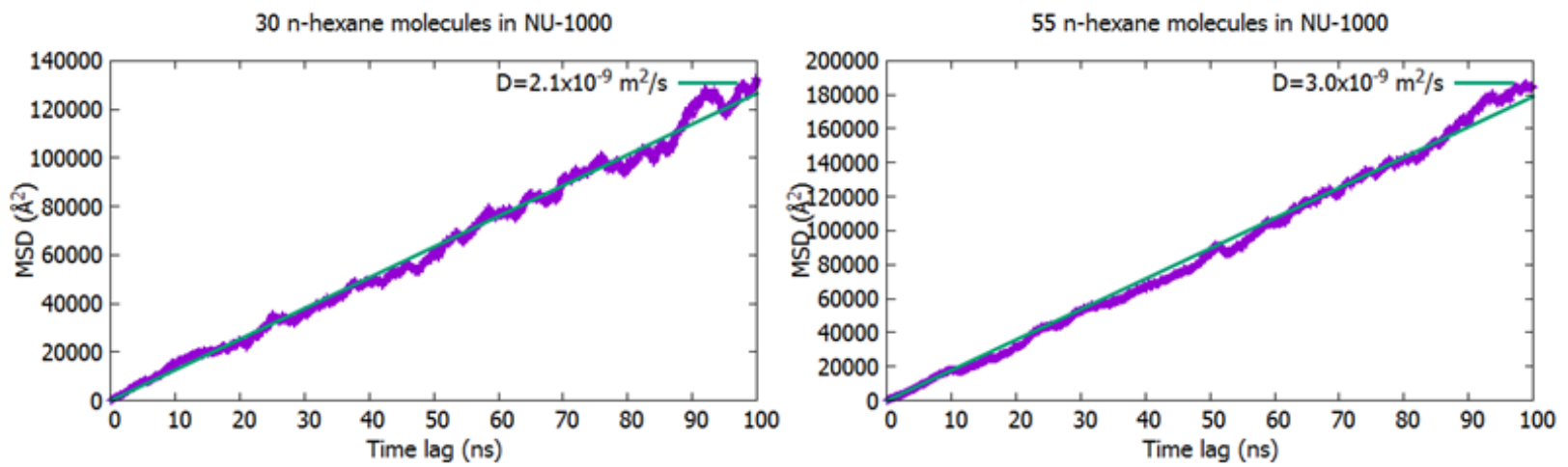

$100 \mathrm{n}$-hexane molecules in NU-1000

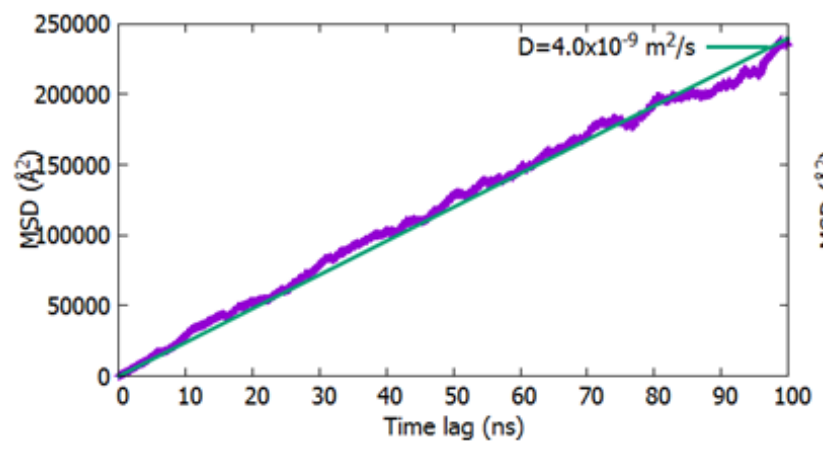

200 n-hexane molecules in NU-1000

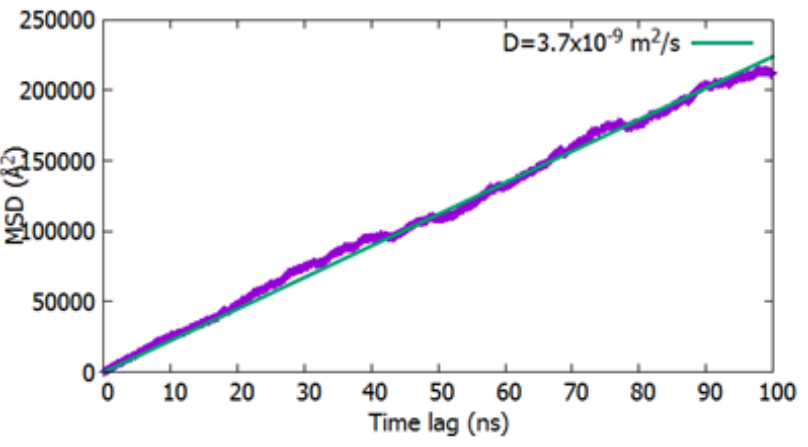

$330 \mathrm{n}$-hexane molecules in NU-1000

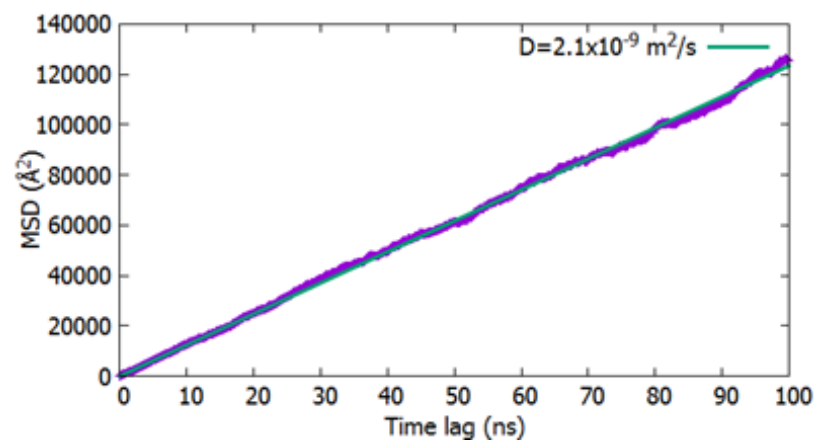

Figure S5. MSD plots of the runs at higher loadings (n-hexane in NU-1000 at $363 \mathrm{~K}$ ). The linearity improves as the loading increases. 


\section{Binding Energy of n-Hexane in the MOFs}

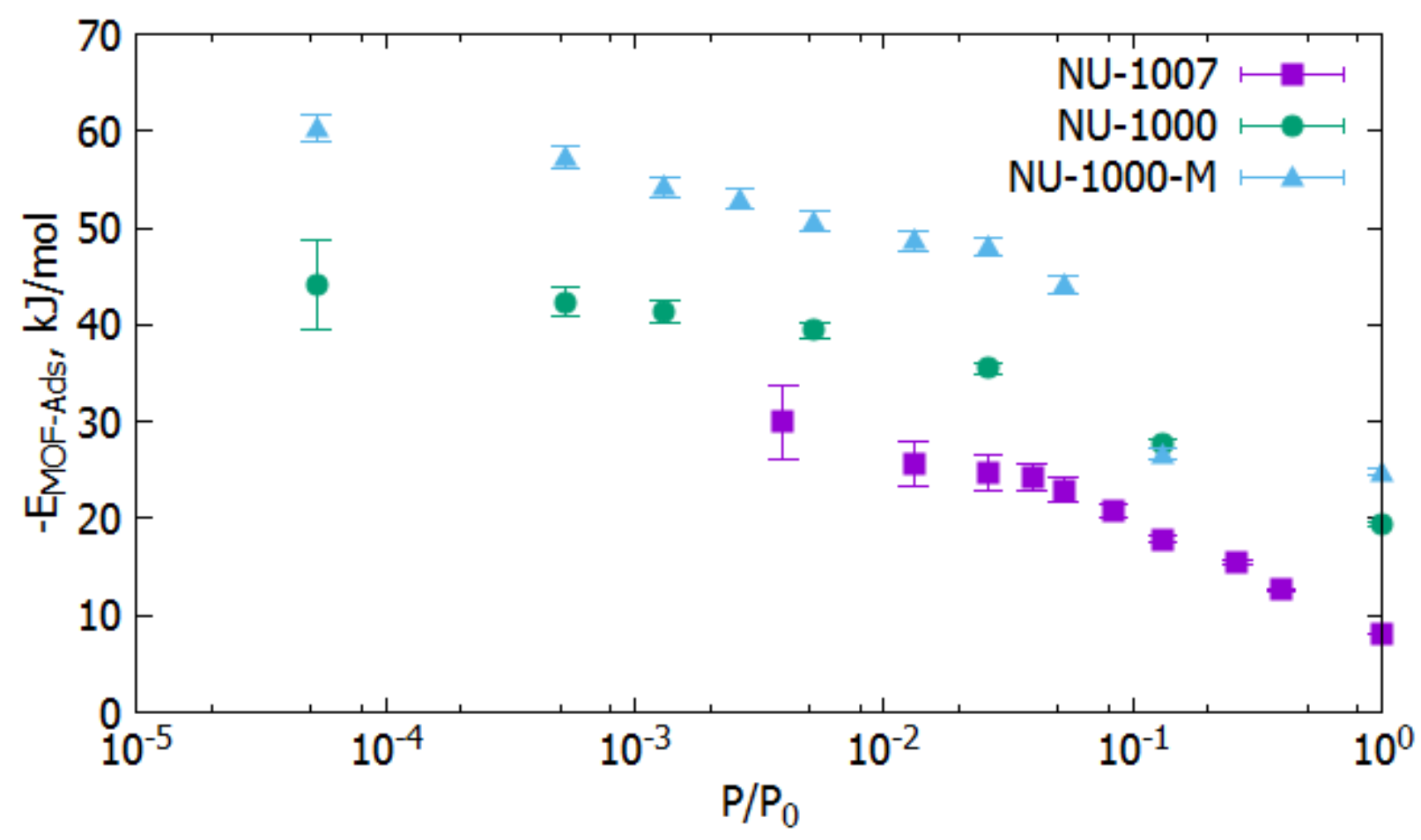

Figure S6. Average binding energy per molecule of n-hexane in NU-1007, NU-1000 and NU-1000-M at $363 \mathrm{~K}$ and different pressures. Average values and error bars were calculated from the results of three simulations for each point. The binding strength decreases in magnitude with increasing pressure as the strongest binding sites get occupied first. 


\section{Single Molecule Trajectory}

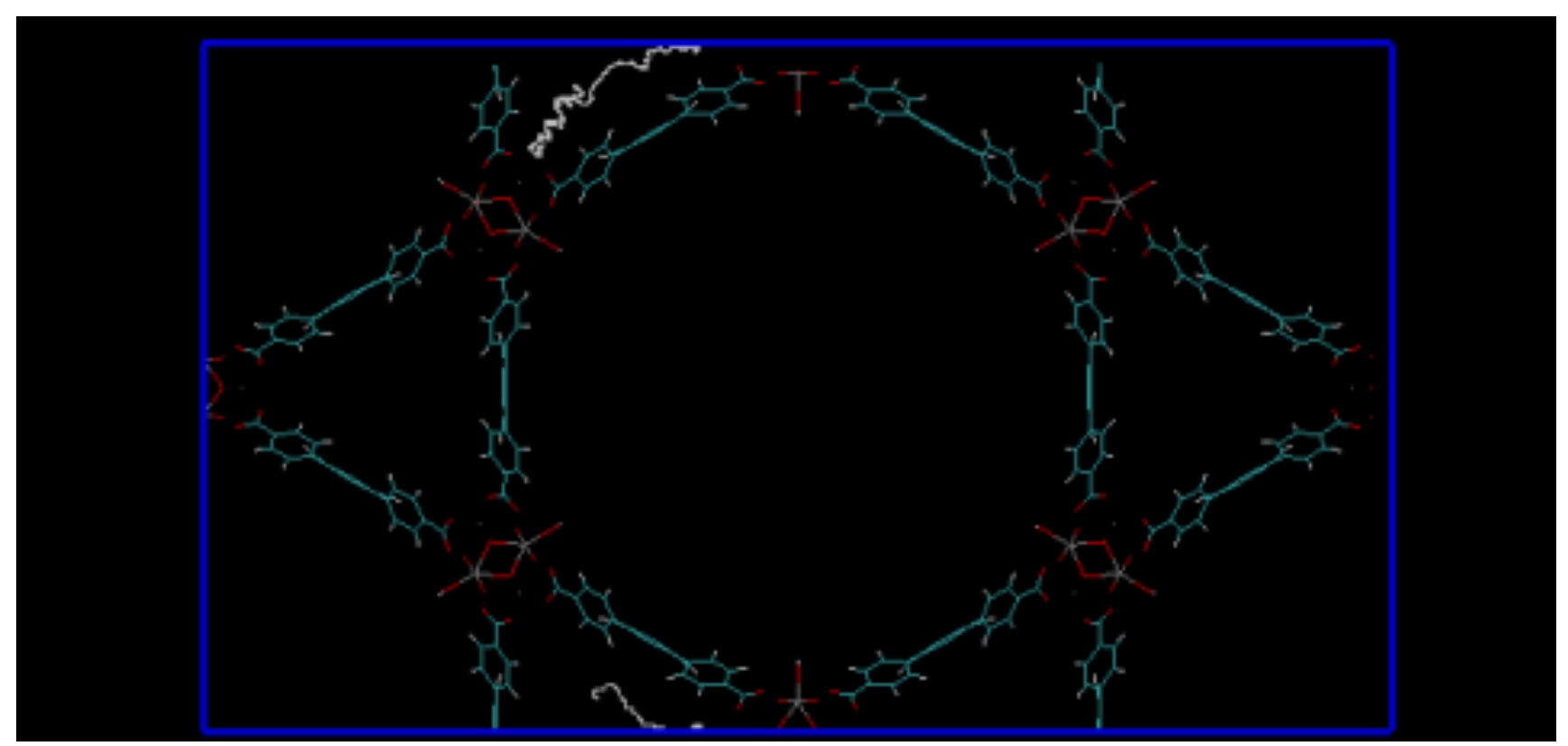

Figure S7. Trajectory of a single n-hexane molecule in NU-1000 at $363 \mathrm{~K}$ from $100 \mathrm{~ns}$ of MD simulation, in which the molecule stays in the microchannel for the entire time. The "discontinuity" in the trajectory is a result of the periodic boundary condition. 
Adsorption Isotherms with Log-X Axis

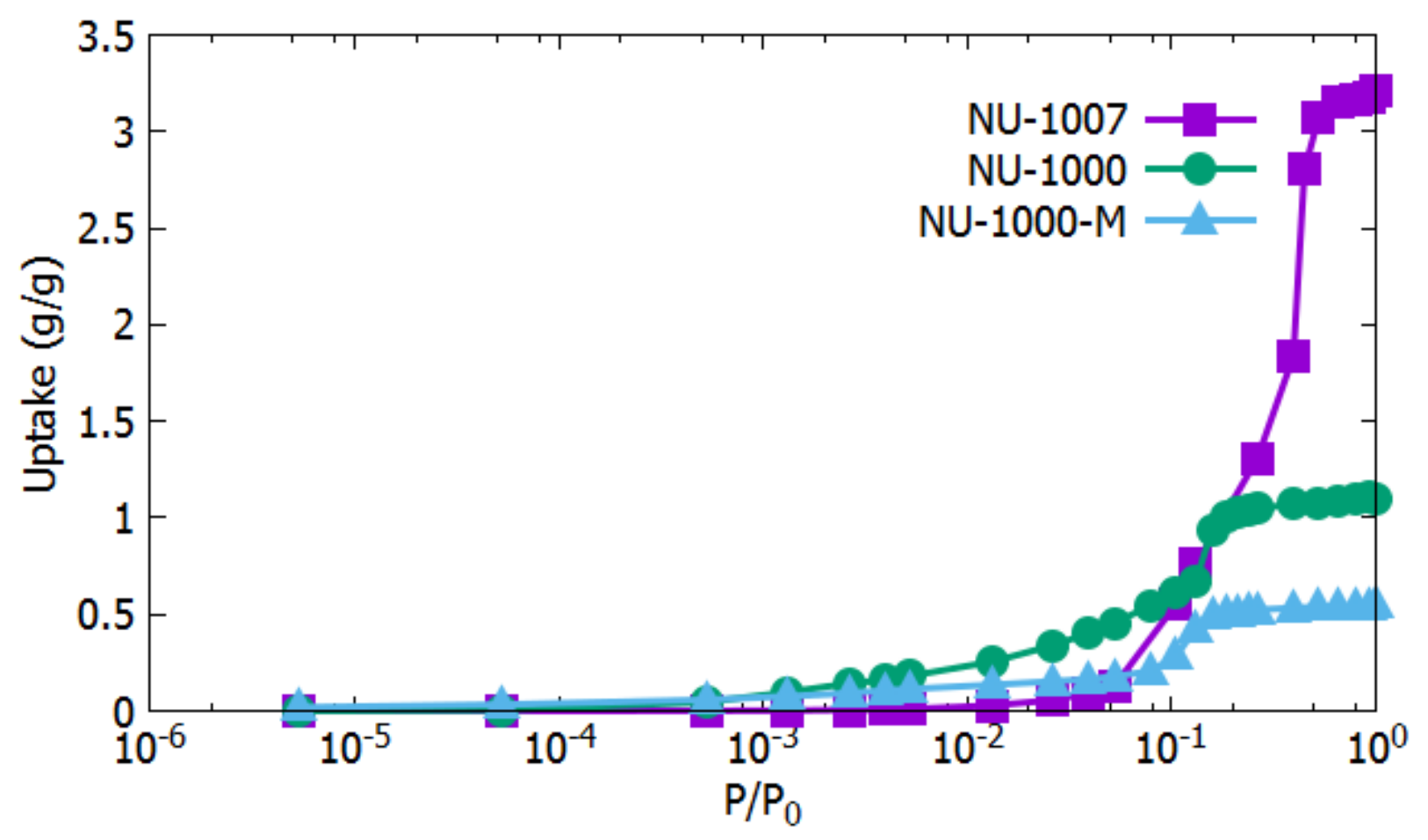

Figure S8. Replot of Figure 2 with $\log$-x axis. 